\title{
Um Modelo de Fórum de Discussão com Suporte às Interações entre Aprendizes utilizando Mapas Conceituais
}

\author{
Fernanda Josirene de Melo Ferreira, Sunny Kelma Oliveira Miranda, Evandro de \\ Barros Costa, Fábio Paraguaçu Duarte da Costa, Hemilis Joyse Barbosa Rocha
}

\author{
Instituto de Computação - Universidade Federal de Alagoas (UFAL) \\ Campus A. C. Simões - CEP:57072-900 - Maceió - AL - Brasil \\ fynanda@gmail.com, sunny@nti.ufal.br, \\ \{evandro, paragua, hjbr\}@ic.ufal.br
}

\begin{abstract}
This article addresses a problem found in Discussion Forums of Collaborative Systems with respect to little investment in the mechanisms that encourage the interaction among learners, considering the low frequency of interaction among them, if compared to level of interaction with the mediator, role played by tutor. To mitigate this problem, we propose in this paper a forum model based on Concept Maps endowed protocols to control the interactions and to support the negotiation in the presence of disagreement. This model was evaluated through a simulation using Colored Petri nets, where we have shown that learner prevailed as focus of interactions, decreasing the hierarchical position of the tutor.
\end{abstract}

Resumo. Este artigo aborda um problema em Fóruns de Discussão dos Sistemas Colaborativos referente ao pouco investimento em mecanismos que favoreçam a interação entre aprendizes, tendo em vista o baixo nível de frequência de interação entre eles, se comparado ao nível de interação com o mediador, papel exercido pelo tutor. Para atenuar tal problema, propôs-se um modelo de fórum baseado em Mapas Conceituais dotado de protocolos para controlar as interações e dar suporte à negociação na presença de desacordos. Avaliou-se tal modelo por meio de uma simulação em rede de Petri Colorida, onde se mostrou que prevaleceu o aprendiz como foco das interações, diminuindo a posição hierárquica do tutor.

\section{Introdução}

Este trabalho está inserido na temática de Sistemas Colaborativos, focado em processos de interação envolvendo aprendizes através da ferramenta assíncrona Fórum de Discussão Educacional. Assume-se aqui que a noção de interação é a base para viabilizar processos de colaboração e sua ausência, portanto, pode comprometer a qualidade da discussão do fórum. O fórum, embora sendo uma das ferramentas mais utilizadas nos ambientes virtuais de aprendizagem que se presta a viabilizar a interação entre os atores envolvidos (professores, tutores e aprendizes), ainda apresenta algumas limitações importantes, a exemplo dos baixos níveis de interação entre aprendizes, tais como discutidos nos estudos mencionados a seguir.

O estudo de Andriola e Loureiro (2005, p. 03) constatou um fórum que "das 144 mensagens trocadas, aproximadamente $91 \%$ deram-se entre um integrante do grupo e o 
professor, sendo praticamente $2 \%$ de mensagens trocadas entre pares de aprendizes". Jacobsohn e Fleury (2005, p. 73) apuraram resultados similares: "do total de 434 mensagens enviadas pelos alunos, apenas $11 \%$ eram respostas ao comentário de um colega; o restante limitou-se à inclusão de uma opinião, uma vez que a participação tinha caráter obrigatório". Da mesma forma, Pereira (2011, p. 357) verificou que os aprendizes ao participarem do fórum "ignoravam as possibilidades de interação com os outros participantes", pois foi prevalecida (p. 360) "a supremacia de mensagens verticalizadas: tanto do modo professora-aluno como ao contrário". Corroboram, ainda, com essas ideias, os trabalhos de Machado e Teruya (2009), Nandi et al (2012) e Tortoreli e Gasparin (2012). Além disso, a pesquisa de Schrum e Benson (2000, p. 56) relata que os aprendizes respondiam as perguntas do professor "sem ler as respostas dos colegas, resultando em comentários lineares ao invés de uma discussão". Assim, é possível concluir que no fórum persiste o modelo da aprendizagem não colaborativa, pois são poucas as interações entre os aprendizes.

Como trabalhos correlacionados à presente pesquisa foram analisados os de Herrera e Fuller (2005), Fucks et al (2005) e Rittgen (2007). O trabalho de Herrera e Fuller integrou mapas conceituais em sua ferramenta assíncrona, mas diferente de fórum. O trabalho de Fucks et al (2005) explorou um fórum, onde há um aprendiz que exerce a função de líder, que abre um tópico e três questões relacionadas ao tema. Ressalta-se que essas questões são lançadas aleatoriamente, sem considerar as contra-argumentações recebidas, deixando os aprendizes sem um feedback de aceitação ou discordância e não provocando desacordos. Já o trabalho de Rittgen (2007) gerou protocolos de negociação para um processo de modelagem colaborativa.

Baseando-se nas constatações sobre características dos trabalhos acima, um problema importante foi levantado: de que forma viabilizar um fórum de discussão que diminua o papel hierárquico do tutor e leve os aprendizes a discutirem com mais frequência entre si? Para abordar esta questão, foi idealizado um modelo de fórum com mecanismos para reduzir a dependência das interações do tutor e que os aprendizes não desvalorizem a leitura das postagens de seus pares. Assim, foi proposto um modelo de fórum baseado em Mapas Conceituais, dotado de protocolos para controlar as interações e dar suporte à negociação na presença de desacordos. Este modelo foi avaliado por meio de simulação, usando-se um método formal com o uso de rede de Petri Colorida. Assim, verificaram-se diferentes cenários de interações colaborativas e negociadas entre os aprendizes, desse modo reduzindo significativamente a interferência do tutor.

\section{Modelo Proposto para Interação em Fórum}

O fórum em pauta permite representar o conhecimento por meio de mapas conceituais a partir de interações entre tutor e aprendizes (autores e colaboradores). O aprendiz deve argumentar o raciocínio que o fez construir seu mapa, podendo adicionar informações que reforcem suas razões para aquele argumento, visto a necessidade de o mapa conceitual ser explicado por um texto complementar (Moreira e Masini (1982)).

\subsection{Protocolos de Interação}

O tutor inicia o fórum descrevendo o tema a ser discutido e seus conceitos relacionados, que devem ser trabalhados individualmente em um mesmo tópico do fórum, como apresentado no protótipo da Figura 1. O primeiro aprendiz a participar de cada conceito 
é denominado autor do mapa conceitual. Os demais aprendizes são denominados do tipo colaborador, os quais escolhem um mapa conceitual de um aprendiz autor para contribuir. Dentro do possível, o sistema estipula a mesma quantidade mínima de aprendizes (não repetidos) do tipo colaborador para cada conceito. Nada impede que um determinado aprendiz que já tenha colaborado com determinado conceito colabore com outro, ou que o aprendiz autor atue como aprendiz colaborador em outro conceito.

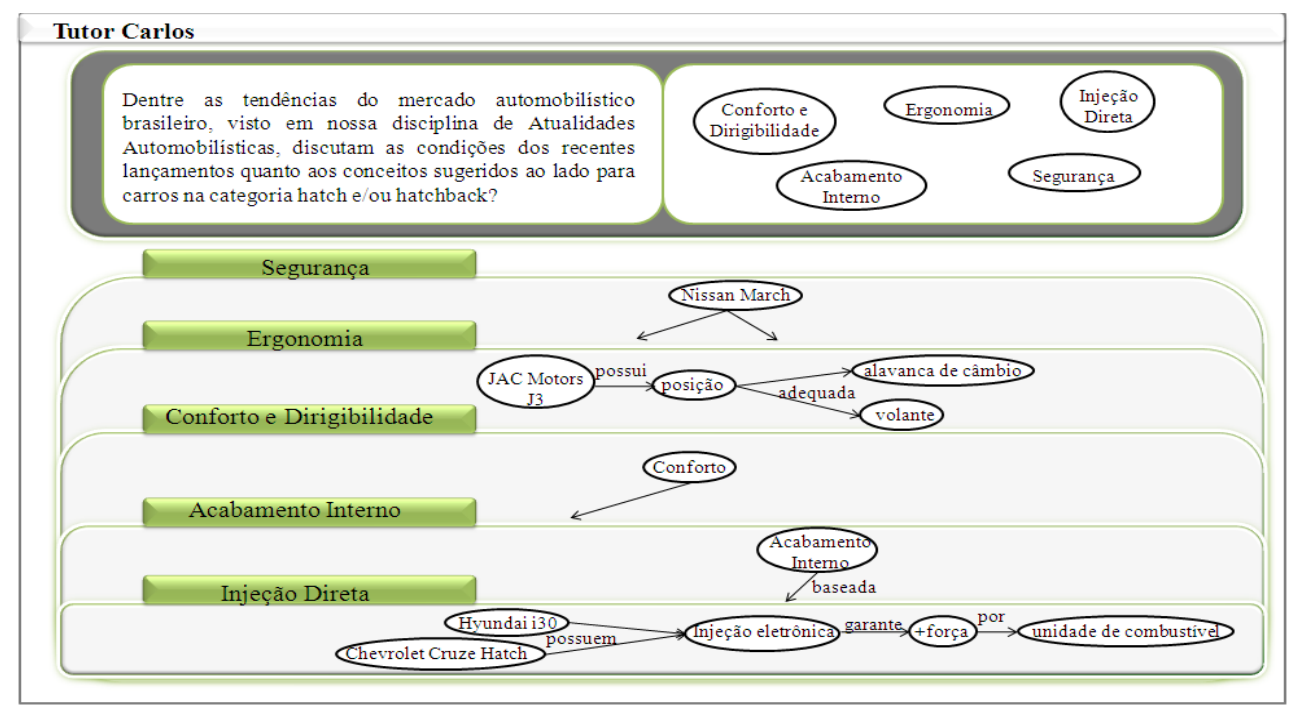

Figura 1. Tela de Conceitos do Aprendiz Autor

\subsection{Protocolos de Negociação}

Rittgen (2007) sugere que os usuários devem interagir por meio de protocolos expressos por ações. Essas ações no fórum proposto foram definidas como "argumentar", "contraargumentar" e "contraproposta" e devem obedecer a uma sequência de acordo com o tipo de aprendiz, conforme a Figura 2.

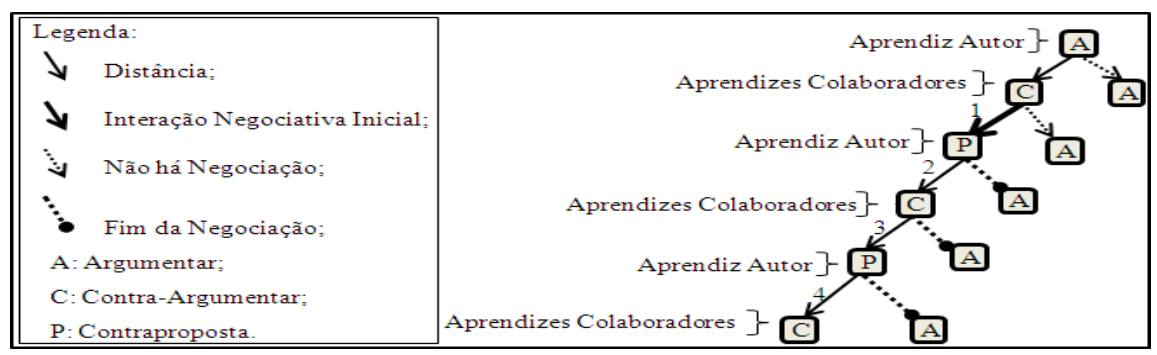

Figura 2. Seqüência de Ações

A interação no fórum é iniciada por meio da ação "argumentar" realizada pelo primeiro usuário a colaborar com a postagem do tutor, tornando-se o aprendiz autor do mapa conceitual. Em seguida, são disponibilizadas para cada aprendiz colaborador duas ações: "contra-argumentar" e "argumentar". Diante das interações recebidas, o fórum disponibiliza ao aprendiz autor as ações: "contraproposta" e "argumentar".

Note que na Figura 2 as ações "contra-argumentar" e "contraproposta" sendo aceitas, estarão seguidas pela ação "argumentar". Assim, as interações do lado esquerdo denotam que está havendo negociação, enquanto que as interações do lado direito 
denotam a resolução da negociação. Numa interação que não seja necessária negociação, ou seja, quando os aprendizes apenas colaborarem sem que haja desacordos, eles farão o uso apenas da ação "argumentar". Logo, a ocorrência dessa ação é contínua até que seja interrompida por um desacordo através da ação "contra-argumentar", mas para as ações "contra-argumentar" e "contraproposta", o sistema não permite a ocorrência sucessiva.

Para casos de impasse na discussão, definiu-se um limite de quatro níveis de interações, que incluem duas "contrapropostas" e três "contra-argumentar", a contar da "Interação Negociativa Inicial" como indicada na figura 2. Se dentro desse limite a negociação não entrar em um consenso, o tutor é convocado para mediar. Caso este não consiga, ele convoca o professor conciliador. $\mathrm{O}$ aprendiz pode desejar contestar uma "argumentação", fruto de um consenso, e assim iniciar uma nova negociação. Para isso, são levados em consideração os mesmos critérios de tolerância, porém a contagem é iniciada a partir dessa ação "argumentar" contestada.

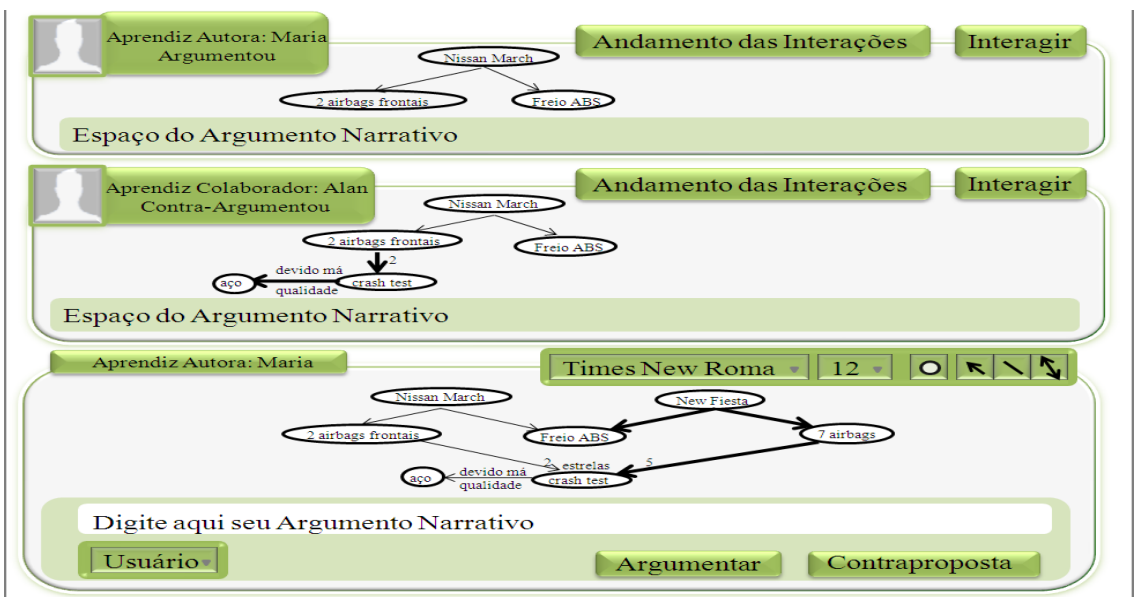

Figura 3. Tela de Interação do Aprendiz Autor em Negociação

Conforme Figura 3, a cada nova interação, o mapa conceitual criado anteriormente é reproduzido no campo de resposta do aprendiz em ação, para que seja possível entendê-lo e expandi-lo. Essa reprodução tenta evitar supostos hábitos dos aprendizes de lerem a postagem do tutor sem responder as respostas dos demais aprendizes. As novas contribuições de cada usuário são identificadas através das relações em negrito em seu campo de resposta. O aprendiz "autor" tem o direito de apagar parte ou todo mapa conceitual para evitar sobrecarga, enquanto o aprendiz "colaborador" tem acesso apenas a mover os conceitos para melhor organizar o mapa.

\subsection{Representação do Modelo de Fórum}

O modelo proposto foi descrito por meio de uma rede de Petri Colorida (Jensen, 1997), visando validação dos protocolos de interação e de negociação mencionados. Essa rede foi desenvolvida por meio da ferramenta CPN Tools ${ }^{2}$ e seu conjunto de cores foi definido por meio das declarações ${ }^{3}$, como expresso na Figura 4. A rede de Petri construída está apresentada na Figura 5, sendo composta de oito lugares ("Tutor", "P1-

\footnotetext{
${ }^{1}$ Exceto para diferentes aprendizes "colaboradores".

${ }^{2}$ Maiores Informações da ferramenta CPN Tools: < http://wiki.daimi.au.dk/cpntools/_home.wiki $>$.

${ }^{3}$ As declarações definem os tipos de dados existentes e as cores das fichas.
} 
n”, "P2-a", "P3-b", “P4-c", "P5-d”, "P6-e”, "Finish") e sete transições (“Autor", "UA", "AA", "C", "AAA", "CC", "Mediation"). Os protocolos do fórum são representados pelos números que indicam ações: $n^{\circ} 1$ - "contra-argumentar", $n^{\circ} 2$ - "argumentar", e no 3 - "contraproposta". Foram definidas sete posições de variáveis (i,n,a,b,c,d,e) que representam a sequência das ações dos protocolos de discussão, com exceção da variável "i”, que guarda as informações iniciais enviadas pelo tutor.

A rede inicia com a disponibilidade de 800 fichas $^{4}$ da variável "ij" presentes no lugar "Tutor". O lugar "Tutor" transfere a variável ("i”) para a transição "Autor", que por sua vez envia uma ficha ao lugar "P1-n" com as sete posições: $(i, 2,6,6,6,6,6)$. Essa ficha indica que o aprendiz "autor" iniciou a interação por meio da ação $\mathrm{n}^{0} 2$ "argumentar" e que as demais posições representadas pelo $\mathrm{n}^{\circ} 6$ estão vazias e poderão ser atualizadas a cada interação. Em seguida, os elementos dessa ficha são endereçados pelas variáveis $(\mathrm{i}, \mathrm{n}, \mathrm{a}, \mathrm{b}, \mathrm{c}, \mathrm{d}, \mathrm{e})$, que serão manipuladas a cada passagem de transição, sendo a primeira, a transição "UA". Em regra geral, na transição "UA" ocorre a interação dos aprendizes do tipo "colaborador" ", e como exceção, a interação do aprendiz "autor" 7 nos seguintes casos de retorno (loop): i) do lugar "P6-e", a partir da segunda posição da ação "argumentar" e nas sucessivas posições pares; ii) das transições "UA", "C" e "CC", a partir da terceira posição da ação "argumentar" e nas sucessivas posições ímpares; iii) da transição "AAA", a partir da quarta posição da ação "argumentar" e nas sucessivas posições pares.

A transição "UA" é responsável por manipular a variável "a" (que representa as ações $n^{\circ} 1$ ou 2). Conforme consta na Figura 4, foi declarada uma variável "p" que receberá um valor inteiro arbitrário entre 1 e 3, representando o arco a ser seguido pela ficha. O primeiro arco ocorre quando a variável "p" é igual a 1, e assim a variável "a" recebe o $\mathrm{n}^{\circ} 1$ - "contra-argumentar" levando a ficha à transição "AA". O segundo arco ocorre quando a variável " $p$ " é igual a 2, e assim, a variável "a" assume o $n^{\circ} 2$ "argumentar", levando a ficha a estacionar na rede, indicando a ausência de desacordo e falta de aprofundamento da discussão. O terceiro arco ocorre quando a variável "p" é igual a 3, que se refere a um retorno ao lugar "P1-n" (ver 1 a Regra Geral da Rede - RGR abaixo). A seguir são apresentadas regras a fim de generalizar algumas ocorrências na rede. Para isso, foi declarada a variável " $\mathrm{t}$ " que percorrerá aleatoriamente por 5 arcos.

1a RGR: Todos os retornos da rede ao lugar "P1-n" foram modelados para não acumularem o $\mathrm{n}^{\mathrm{o}} 2$ ("argumentar") e, sim, serem substituídos e alocados na posição da variável "n", necessário para garantir que as próximas 5 vagas $(a, b, c, d, e)$ estejam disponíveis para uma possível interação negociada entre aprendizes do tipo "colaborador". Assim os aprendizes terão as mesmas condições (quatro interações) de se chegar a um acordo, antes que seja preciso a interferência do tutor ou professor. Por isso, o resultado final obtido na rede (Figura 5) omite as interações que ocorreram antes da ficha percorrer por um dos retornos (quando $\mathrm{t}=4$ ).

\footnotetext{
${ }^{4}$ As fichas (token) que percorrem na rede representam um conjunto de interações dentro de um conceito.

${ }^{5}$ Variáveis são objetos obtidos a partir de declarações, que permitem a manipulação dos dados da rede.

${ }^{6} \mathrm{O}$ aprendiz "colaborador" também é representado pelas transições "C" e "CC".

${ }^{7} \mathrm{O}$ aprendiz "autor" também é representado pelas transições "AA" e "AAA".
} 
O loop da ação 2 - "argumentar" terá fim assim que ocorra um indício de uma negociação entre aprendizes do tipo "colaborador", ou seja, com a presença da ação 1 "contra-argumentar" na posição da variável "a" e tratada ainda pela transição "UA". Quando a variável "a" ultrapassar a condição da transição "AA", ou seja, ser diferente de 2 , a próxima variável "b" estará apta para ser manipulada. Um retorno de exceção válido ainda para esta regra se refere ao lugar "P6-e" até a transição "UA". Os demais retornos são tratados mais a frente na $5^{\mathrm{a}} \mathrm{RGR}$.

2a RGR: Toda variável " $t$ " igual a 1 , a negociação continua, ou seja, a ficha continua a percorrer pela rede.

$3^{\text {a }}$ RGR: Toda variável " $t$ " igual a 2 é necessária para que a ficha dê continuidade por cada retorno de sua próxima transição para o lugar "P1-n". Essa continuidade se refere à interação colaborativa, pois se a variável trabalhada em cada transição receber o n 2 - "argumentar", a negociação é finalizada. Ao ocorrer os retornos mencionados, a ação de $\mathrm{n}^{\circ} 2$ poderá entrar em loop (entre o lugar "Pn-1" e a transição "UA"). Esse loop terá fim caso haja uma interação negociada entre aprendizes do tipo "colaborador" quando a ficha conseguir percorrer da transição "UA" para o lugar "P2-a" (sendo p igual a 1) ou, sendo "p" igual a 2, o que indica a última colaboração por meio da ação "argumentar". Pode ocorrer, também, a ficha chegar pela primeira vez na transição "UA", originada da transição "Autor" e parar em "p" igual a 2.

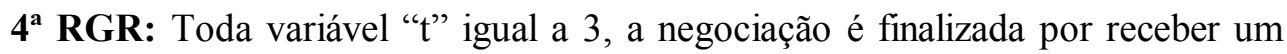
zero após o inteiro 2 - "argumentar". Tanto na $3^{\mathrm{a}} \mathrm{RGR}$, como na presente, a negociação só não é finalizada quando "t" igual a 2 ou quando "t" igual a 3 ambas na transição "AA", respectivamente, pois houve apenas um indício de negociação, mas esta não foi efetivada. Para de fato haver a negociação, a ação 1 - "contra-argumentar" deve ser contestada pela ação 3 - "contraproposta".

$5^{\text {a }}$ RGR: Toda variável " $t$ " igual a 5 foi modelada de forma parecida a $1^{\text {a }}$ RGR: não acumulando o $n^{\circ} 1$ - "contra-argumentar". Essa regra exerce a função referente à necessidade do $\mathrm{n}^{\mathrm{o}} 1$ ocorrer continuamente, desde que utilizado por diferentes aprendizes do tipo "colaborador" perante a proposta do aprendiz "autor". Identifica-se isso na rede pela terminação de $\mathrm{n}^{\mathrm{o}} 8 \mathrm{em}$ seus retornos. Com exceção do retorno de "Mediation" para "P6-e", que termina com $\mathrm{n}^{\mathrm{o}} 1$ devido à falta de lugar na ficha por estar no limite da negociação, isto é, prestes ao tutor/professor interromper a discussão afim de consenso.

Na Figura 5, os resultados provenientes da simulação do modelo são observados nos retângulos em cor verde. Resultados com valores das variáveis terminados em 6 , indicam que a interação se deu entre o aprendiz do tipo "autor" e diferentes "colaboradores". Resultados com valores das variáveis terminados em 9, indicam que antes do indício ou de iniciar a interação negociada entre aprendizes do tipo "colaborador" houve interações colaborativas. No caso da presença do $\mathrm{n}^{\mathrm{o}} 0$, antes de uma seqüência ou unidade de $n^{\circ} 6,9$ ou 8 , indica que a negociação foi resolvida sem que fosse preciso preencher as demais variáveis com novos valores de ações do protocolo.

Por fim, a transição "Mediation" é habilitada caso os aprendizes não consigam entrar em um consenso, sendo preciso a interferência do tutor ou do professor. 

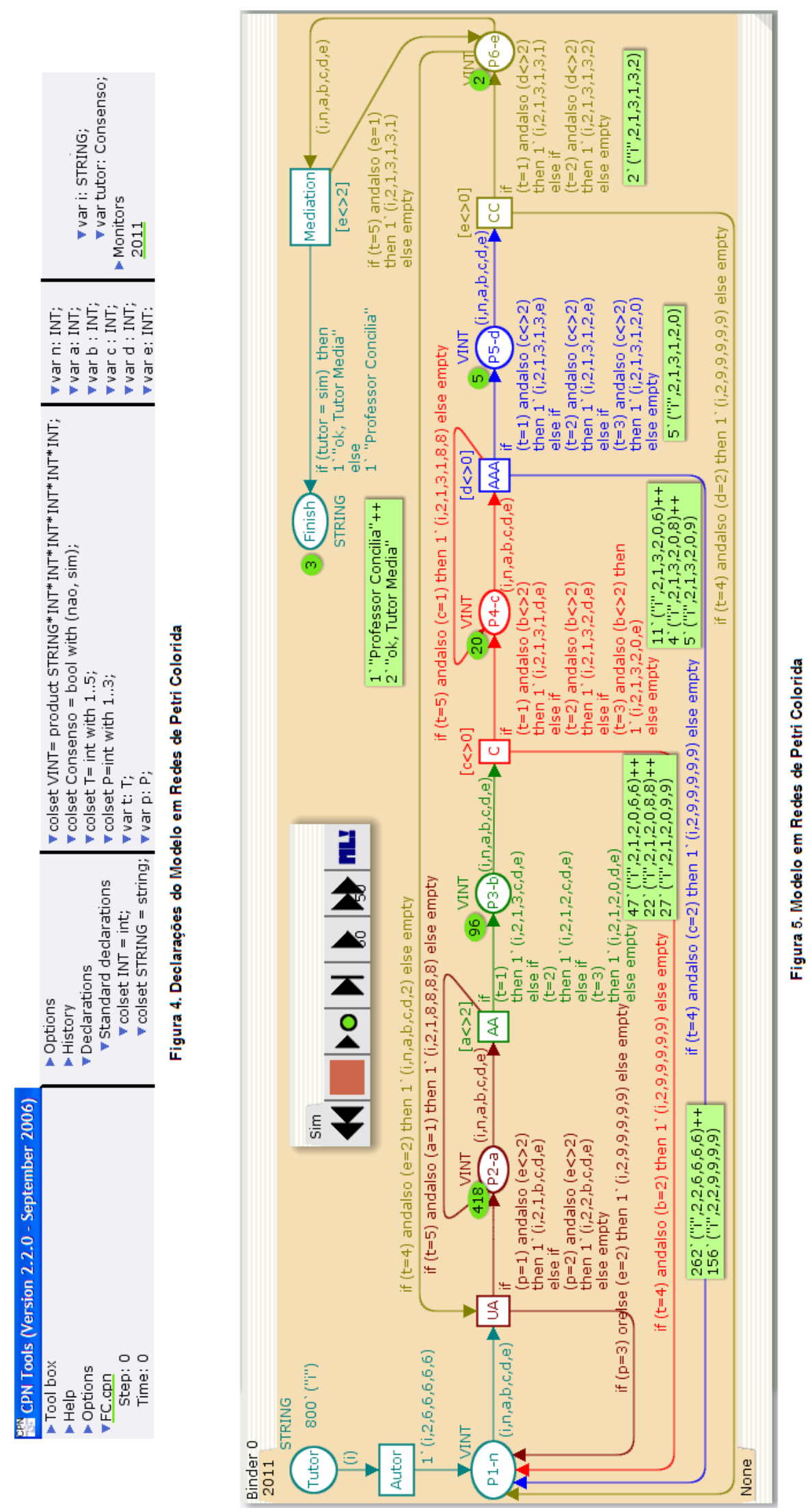


\section{Análise dos Resultados e Discussões}

Analisando os resultados possíveis e expostos na rede de Petri Colorida (Figura 5), obtêm-se o Quadro 1. Considere "A" para a ação de $\mathrm{n}^{\circ} 2$ - "argumentar", "C" para a ação de no 1 - "contra-argumentar" e "P" para a ação de n 3 - "contraproposta".

Quadro 1. Análise dos Resultados da RPC

\begin{tabular}{|c|c|c|c|c|c|c|c|c|c|c|}
\hline \multirow[b]{3}{*}{ Indicativo } & & \multicolumn{6}{|c|}{ Aprendizes } & Loop & \multicolumn{2}{|c|}{ Considerações } \\
\hline & $\stackrel{\check{\Xi}}{\Xi}$ & | & 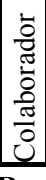 & 泀 & \begin{tabular}{l|} 
\\
$\frac{0}{\pi}$ \\
$\frac{\pi}{0}$ \\
$\frac{\pi}{0}$ \\
\\
\end{tabular} & 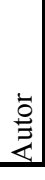 & 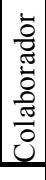 & \multirow[t]{2}{*}{ Ação } & \multirow{2}{*}{\multicolumn{2}{|c|}{$\begin{array}{l}\text { Suponha que, para todos os resultados obtidos na rede, o Tutor } \\
\text { tenha enviado as informações iniciais "i" para abrir o tópico do } \\
\text { fórum. Dessa forma, os aprendizes já têm conhecimento do } \\
\text { assunto a ser discutido. Considere, ainda, que o aprendiz "autor" } \\
\text { tenha iniciada a discussão, indicada pela ação n } \text { n }^{\circ} \text { - "argumentar". }\end{array}$}} \\
\hline & \multicolumn{7}{|c|}{ Resultados } & & & \\
\hline \multirow{4}{*}{$\begin{array}{c}\text { Interações } \\
\text { Colaborativas }\end{array}$} & $\mathrm{i}$ & 2 & 2 & 6 & 6 & 6 & 6 & \multirow{4}{*}{$\begin{array}{l}\text { Sim, } \\
\text { A. }\end{array}$} & \multirow{2}{*}{\multicolumn{2}{|c|}{$\begin{array}{l}\text { Resultado não desejável. Interação somente de um par de } \\
\text { aprendizes, indicada pela terminação de } n^{\circ} 6 \text {. }\end{array}$}} \\
\hline & & $\mathrm{A}$ & $\bar{A}$ & - & - & - & - & & & \\
\hline & $\bar{i}$ & 2 & 2 & 9 & 9 & 9 & 9 & & \multirow{2}{*}{\multicolumn{2}{|c|}{$\begin{array}{l}\text { Houve incontáveis interações dos aprendizes, indicada pela } \\
\text { terminação de } \mathrm{n}^{\circ} 9 \text {. }\end{array}$}} \\
\hline & & $\mathrm{A}$ & $\bar{A}$ & - & - & - & - & & & \\
\hline \multirow{6}{*}{$\begin{array}{c}\text { Indício de } \\
\text { Negociação }^{8}\end{array}$} & $\mathrm{i}$ & 2 & 1 & 2 & 0 & 6 & 6 & \multirow{2}{*}{ Não. } & \multirow{6}{*}{$\begin{array}{l}\text { O aprendiz "colaborador" } \\
\text { interage por meio da ação } \\
\text { "contra-argumentar". Em } \\
\text { seguida, o aprendiz "autor" } \\
\text { concorda com os contra } \\
\text { argumentos recebidos e } \\
\text { contribui ainda mais, entrando } \\
\text { em consenso pela ação } \\
\text { "argumentar". }\end{array}$} & \multirow{2}{*}{$\begin{array}{l}\text { Resultado razoável. Poucas } \\
\text { interações (apenas três). }\end{array}$} \\
\hline & & $\mathrm{A}$ & $\mathrm{C}$ & $\mathrm{A}$ & - & - & - & & & \\
\hline & $\mathrm{i}$ & 2 & 1 & 2 & 0 & 8 & 8 & \multirow[b]{2}{*}{$\begin{array}{l}\text { Sim, } \\
\text { C. }\end{array}$} & & \multirow{2}{*}{$\begin{array}{l}\text { Incontáveis interações dos } \\
\text { aprendizes "colaboradores" por } \\
\text { meio da ação "C", indicado pelo } \\
\mathrm{n}^{\circ} 8 \text {. }\end{array}$} \\
\hline & & A & $\mathrm{C}$ & $\mathrm{A}$ & - & - & - & & & \\
\hline & $\mathrm{i}$ & 2 & 1 & 2 & 0 & 9 & 9 & \multirow{2}{*}{$\begin{array}{l}\text { Sim, } \\
\text { A. }\end{array}$} & & \multirow{2}{*}{$\begin{array}{l}\text { Incontáveis interações } \\
\text { aprendizes por meio da ação } \\
\text { "A", indicado pelo } \mathrm{n}^{\circ} 9 .\end{array}$} \\
\hline & & $\mathrm{A}$ & $\mathrm{C}$ & $\mathrm{A}$ & - & - & - & & & \\
\hline \multirow{10}{*}{$\begin{array}{l}\text { Interação } \\
\text { Negociada }\end{array}$} & 1 & 2 & 1 & 3 & 2 & 0 & 6 & \multirow{2}{*}{ Não. } & \multirow{6}{*}{ 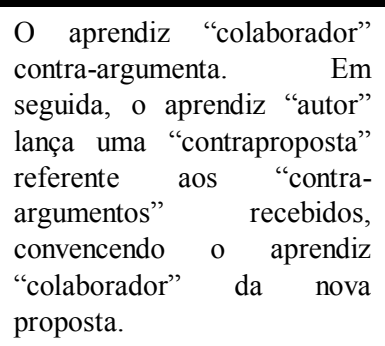 } & \multirow{2}{*}{$\begin{array}{l}\text { Resultado razoável. Poucas } \\
\text { interações (apenas quatro). }\end{array}$} \\
\hline & & $\mathrm{A}$ & $\mathrm{C}$ & $\mathrm{P}$ & $\mathrm{A}$ & - & - & & & \\
\hline & $\mathrm{i}$ & 2 & 1 & 3 & 2 & 0 & 8 & \multirow[b]{2}{*}{$\begin{array}{l}\text { Sim, } \\
\text { C. }\end{array}$} & & \multirow{2}{*}{$\begin{array}{l}\text { Incontáveis interações dos } \\
\text { aprendizes "colaboradores" por } \\
\text { meio da ação "C", indicado pelo } \\
n^{\circ} 8 \text {. }\end{array}$} \\
\hline & & A & $\mathrm{C}$ & $\mathrm{P}$ & $\mathrm{A}$ & - & - & & & \\
\hline & $\mathrm{i}$ & 2 & 1 & 3 & 2 & 0 & 9 & \multirow{2}{*}{$\begin{array}{l}\text { Sim, } \\
\text { A. }\end{array}$} & & \multirow{2}{*}{$\begin{array}{l}\text { Incontáveis interações } \text { dos } \\
\text { aprendizes por meio da ação } \\
\text { "A", indicado pelo n } 9 .\end{array}$} \\
\hline & & A & $\mathrm{C}$ & $\mathrm{P}$ & $\mathrm{A}$ & - & - & & & \\
\hline & $\mathrm{i}$ & 2 & 1 & 3 & 1 & 2 & 0 & & \multirow{6}{*}{$\begin{array}{l}\text { O aprendiz "colaborador" } \\
\text { interage por meio da ação } \\
\text { "contra-argumentar" e, depois } \\
\text { recebe uma "contraproposta" } \\
\text { do aprendiz "autor". Por não } \\
\text { concordar, ele envia nova } \\
\text { ação "contra-argumentar". }\end{array}$} & \multirow{2}{*}{$\begin{array}{l}\text { Aprendiz "autor" aceita, por } \\
\text { meio da ação "A", os contra- } \\
\text { argumentos recebidos. }\end{array}$} \\
\hline & & $\mathrm{A}$ & $\mathrm{C}$ & $\mathrm{P}$ & $\mathrm{C}$ & A & - & & & \\
\hline & $\mathrm{i}$ & 2 & 1 & 3 & 1 & 3 & 2 & & & \multirow{2}{*}{$\begin{array}{l}\text { Aprendiz "autor" rejeita, por } \\
\text { meio da ação "P", os contra- } \\
\text { argumentos recebidos. Por fim, o } \\
\text { aprendiz "colaborador" } \\
\text { concorda, por meio da ação "A". }\end{array}$} \\
\hline & & $\mathrm{A}$ & $\mathrm{C}$ & $\mathrm{P}$ & $\mathrm{C}$ & $\mathrm{P}$ & A & 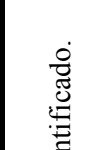 & & \\
\hline \multirow[b]{2}{*}{$\begin{array}{l}\text { Negociação } \\
\text { sem } \\
\text { Consenso: } \\
\text { "Ok, Tutor } \\
\text { Media" e } \\
\text { "Professor } \\
\text { Concilia" }\end{array}$} & 1 & 2 & 1 & 3 & 1 & 3 & 1 & \multirow{2}{*}{ 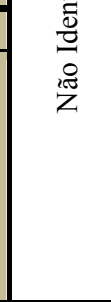 } & & Aprendiz "autor" rejeita, por \\
\hline & & $\mathrm{A}$ & $\mathrm{C}$ & $\mathrm{P}$ & $\mathrm{C}$ & $\mathrm{P}$ & $\mathrm{C}$ & & & $\begin{array}{l}\text { meio da ação "P", os contra- } \\
\text { argumentos recebidos. Aprendiz } \\
\text { "colaborador" discorda da nova } \\
\text { proposta, por meio da ação "C", } \\
\text { gerando impasse, sendo } \\
\text { necessária a convocação do } \\
\text { Tutor/Professor. }\end{array}$ \\
\hline
\end{tabular}

\footnotetext{
${ }^{8}$ Indício de Negociação, mas não efetivada, pois a ação “contra-argumentar” foi aceita.
} 
Das considerações constatadas no Quadro 1, observa-se que a maioria dos resultados analisados foram satisfatórios, restando apenas três situações em que houve pouca interação. Os resultados favoráveis apontaram interações que contribuíram para uma maior discussão entre os aprendizes, colocando-os no foco da discussão e tornandoos independentes da atuação constante do tutor, que teve sua posição hierárquica diminuída.

Da simulação da rede (Figura 5) observou-se que das 800 fichas disponíveis, somente três delas geraram situações de impasse nas discussões entre os aprendizes, sendo necessária a convocação do tutor ou professor. Nessas situações, o tutor mediador solucionou por duas vezes, e apenas uma vez ficou a cargo do professor conciliador. Isso demonstrou baixa sobrecarrega das atividades do tutor/professor, resultado da maior liberdade oferecida ao aprendiz, sobretudo ao aprendiz "autor" que exerce papel de líder de um conceito do tema discutido.

Portanto, esses resultados contribuíram na compreensão das possíveis interações que podem ocorrer no fórum proposto fazendo uso dos protocolos de interação e negociação.

\section{Considerações Finais e Trabalhos Futuros}

Essa pesquisa levantou algumas limitações observadas nas interações do ambiente fórum de discussão, notadamente no que se refere à existência de poucos mecanismos que se prestem a estimular mais interações entre aprendizes, relativamente ao que se observa na maior quantidade de interações entre aprendizes e tutor. Para contribuir na amenização dessas limitações, apresentou um recurso alternativo para ser utilizado no contexto de um fórum: a inserção colaborativa e controlada de artefatos, no caso a construção de mapas conceituais. Desse modo, a dinâmica no fórum passa a funcionar conforme as regras apresentadas nos protocolos de interação e de negociação. A partir destas regras é possível, respectivamente, organizar a distribuição de aprendizes por conceitos e conduzi-los a se comportarem conforme a sequência de ações de protocolos a serem cumpridas e disponibilizadas de acordo com o tipo de usuário aprendiz. O fórum também prevê eventuais desacordos, visto que construir mapas conceituais é idiossincrático. Para isto, foram propostos protocolos de negociação modelados formalmente através da rede de Petri Colorida. Os resultados finais apresentados na simulação deste modelo mostraram de forma concisa a visualização dos tipos de interações no fórum.

Como prolongamento natural desse trabalho, está em andamento um investimento na implementação do modelo do fórum proposto integrado ao Moodle. Em seguida, experimentá-lo em uma turma no sistema UAB, a fim de realizar um estudo comparativo com o fórum de discussão tradicional, para averiguar o nível de aprendizagem e aceitação da nova ferramenta perante os aprendizes.

Outras pesquisas que podem fazer evoluir este trabalho, está na geração automática de um mapa conceitual que represente um feedback correspondente ao tópico do fórum, após o seu término, bem como automatizar a comparação dos mapas e ainda adaptar o fórum conceitual a qualquer outra notação gráfica além do mapa conceitual, como por exemplo, mapas mentais, diagrama de classes, ontologia, etc., a escolha do tutor. 


\section{Referências}

Andriola, W. B. ; Loureiro, R. (2005) Sistematização da avaliação da aprendizagem em comunidades organizadas no ciberespaço. Revista Iberoamericana de Educación (Online), Madrid, v. 37, n. 1, p. 1-5.

Baker, M. J. (1999). Argumentation and Constructive Interaction. G. Rijlaarsdam \& E. Espéret (Series Eds.) \& Pierre Coirier and Jerry Andriessen (Vol. Eds.) Studies in Writing: Vol. 5. Foundations of Argumentative Text Processing, 179 - 202. Amsterdam: University of Amsterdam Press.

Fuks, H. ; Gerosa, M. A. ; Pimentel, M. ; Filippo, D. ; Lucena, C. J. P. (2005) Informações Estatísticas e Visuais para a Mediação de Fóruns Educacionais. Revista Brasileira de Informática na Educação, Florianópolis, v. 13, n.3, p. 19-32.

Herrera, O.; Fuller, D. A. (2005). Shared Knowledge: The Result of Negotiation in Nonhierarchical Environments. Springer Verlag in Lecture Notes of Computer Science (LNCS), vol 3706, pp 255-262.

Jacobsohn, L. V.; Fleury, M. T. L. (2005). A contribuição do fórum de discussão para o aprendizado do aprendiz: uma experiência com estudantes de administração. Caderno de Pesquisas em Administração (USP), São Paulo, v. 12, n.1, p. 69-80.

Jensen, K.. (1997) Coloured Petri Nets: Basic Concepts, Analysis Methods and Practical Use. New York, v. 2, Springer-Verlag.

Machado, S. F.; TERUYA, T. K. (2009) Mediação Pedagógica em Ambientes Virtuais de Aprendizagem: a perspectiva dos alunos. Políticas e Práticas Educativas: desafios da aprendizagem. In: Congresso Nacional de Educação, 9. Curitiba. Anais...Curitiba: EDUCERE, 2009, p. 1726-1739.

Moreira, M. A.; MASINI, E. F. S. (1982) Aprendizagem Significativa: A Teoria de David Ausubel. Editora Moraes.

Nandi, D.; Hamilton, M.; Chang, S.; Balbo, S. (2012). Evaluating quality in online asynchronous interactions between students and discussion facilitators. Australasian Journal of Educational Technology, 28(4), 684-702.

Pereira, S. V. M.. (2011) Interação em fóruns de $\mathrm{EaD}$ a otimização de um espaço de aprendizagem colaborativa. In: Eutomia, v. 1, p. 349-364.

Rittgen, P.. (2007) Negotiating Models. In: Advanced Information Systems Engineering, 19th International Conference, CAiSE 2007, Trondheim, Norway, Proceedings, LNCS 4495, Berlin, Germany: Springer, 2007, pp. 561-573.

Schrum, L.; Benson, A.. (2000) Online Professional Education: a case study of an MBA program through its transition to an online model. Journal of Asynchronous Learning Networks, v. 4, n. 1.

Tortoreli, A. C. ; Gasparin, J. L. . (2012) A interação do professor e alunos no ambiente virtual de aprendizagem: a ferramenta assíncrona fórum. In: Encontro Nacional de Didática e Práticas de Ensino, 16., 2012. Campinas. Anais...Campinas: UNICAMP, p. 23-34. 